\title{
MORPHOLOGICAL CODING OF COLOR IMAGES BY VECTOR CONNECTED FILTERS
}

\author{
Jesús Angulo, Jean Serra \\ Centre de Morphologie Mathématique - Ecole des Mines de Paris \\ 35, rue Saint-Honoré, 77305 Fontainebleau, France; \{angulo,serra\}@ cmm.ensmp.fr
}

\begin{abstract}
This paper deals with the use of the vector levelings for coding color images, a class of morphological connected filters which suppresses details but preserves the contours of the remaining objects. If the color images are filtered by independently leveling each color component, new colors may be introduced. In order to avoid this drawback, a total order must be imposed on the color vectors. A comparative study has been drawn for various lexicographical orders in the RGB and the HLS color systems. These filters can be especially useful as a preprocessing step for improving the compression of color images.
\end{abstract}

\section{INTRODUCTION}

The morphological connected filters have the nice property to suppress details but preserve the contours of the remaining objects. Levelings are a subclass of symmetric connected operators that have originally been defined and studied for grey tone images [7]. Several extensions to vector spaces have been proposed: pseudo-scalar and autarkical levelings [2], separable levelings [8] and non-separable levelings [12]. In this paper, we deal with the implementation of vector levelings in complete totally ordered lattices by using lexicographical orders which are defined on the RGB color space and on an improved HLS system (recently proposed [5]). After a comparative study of the performance of different orders, these color filters are applied to the morphological coding of color images.

\section{MATHEMATICAL MORPHOLOGY IN COLOR COMPLETE TOTALLY ORDERED LATTICES}

Mathematical morphology is a non-linear image processing approach which is based on the application of lattice theory to spatial structures [10]. In practice, the definition of morphological operators needs a totally ordered complete lattice structure $(x \leq y$ or $y \leq x$ for every pair $x$ and $y$; and every finite subset has a supremum and an infimum) [11]: there are no pair of points for which the order is uncertain. The application of mathematical morphology to color images is difficult due to the vectorial nature of the color data. Many research works have been carried out on the application of mathematical morphology to color images [6, 3, 4, 9]. The most commonly adopted approach is based on the use of a lexicographical order which imposes a total order on the vectors. Let $\mathbf{x}=\left(x_{1}, x_{2}, \cdots, x_{n}\right)$ and $\mathbf{y}=\left(y_{1}, y_{2}, \cdots, y_{n}\right)$ be two arbitrary vectors $\left(\mathbf{x}, \mathbf{y} \in Z^{n}\right)$, an example of lexicographical order may be

$$
\mathbf{x}<\mathbf{y} \text { if }\left\{\begin{array}{llll}
x_{1}<y_{1} & \text { or } & & \\
x_{1}=y_{1} & \text { and } & x_{2}<y_{2} & \text { or } \cdots \\
x_{1}=y_{1} & \text { and } & x_{2}=y_{2} \cdots & x_{n}<y_{n}
\end{array}\right.
$$

In this case the priority is given to the first component, then to the second, etc. Obviously, it is possible to define other orders for imposing a dominant role to any other of the vector components. As previous works have shown [9], the drawback of these kinds of orders is that most of vector pairs are sorted by the chosen first component. There is a simple way in order to make the lexicographical order more flexible (reducing the excessive dependence of the first component) which involves the linear reduction of the dynamic margin of the first component, applying a division by a constant and rounding off. Therefore, we can propose an $\alpha$-modulus lexicographical order

$\mathbf{x}<_{\alpha} \mathbf{y}\left\{\begin{array}{llll}\left\lceil x_{1} / \alpha\right\rceil<\left\lceil y_{1} / \alpha\right\rceil & \text { or } & & \\ \left\lceil x_{1} / \alpha\right\rceil=\left\lceil y_{1} / \alpha\right\rceil & \text { and } & x_{2}<y_{2} & \text { or } \cdots \\ \left\lceil x_{1} / \alpha\right\rceil=\left\lceil y_{1} / \alpha\right\rceil & \text { and } & x_{2}=y_{2} \cdots & x_{n}<y_{n}\end{array}\right.$

The choice of the value for $\alpha$ controls the degree of influence of the first component with regard to the others (above all the second one). These orders can be applied to the color spaces. Let $\mathbf{f}(p)=\left(f_{R}(p), f_{G}(p), f_{B}(p)\right)$ and $\mathbf{f}(p)=\left(f_{H}(p), f_{L}(p), f_{S}(p)\right)$ be the color values of the pixel $p$ from the color image $\mathbf{f}$ in the RGB and HLS color spaces respectively.

The use of a lexicographical order directly in the RGB space requires that one of the color must be arbitrarily elevated to a dominant role. To avoid this, a first approach entails calculating the $\max$ and the 
min of the three RGB values for every pixel; i.e $M(\mathbf{f})(p)=\max \left(f_{R}(p), f_{G}(p), f_{B}(p)\right)$ and $m(\mathbf{f})(p)=$ $\min \left(f_{R}(p), f_{G}(p), f_{B}(p)\right)$. Then for every pair of pixels $p$ and $q$ we can build a lexicographical order where the first component is given by $M$, the second one is associated to $m$ and, if $M(p)=M(q)$ and $m(p)=m(q)$ then the choice of the RGB components does not have a significant influence; e.g. the green component can be taken into account then the red and finally the blue $\left(M, m, f_{G}, f_{R}, f_{B}\right)$. We named this order $M m-R G B$. Preliminary tests showed that the application of morphological operators based on the $M m-R G B$ lattice yields strange visual effects. In order to improve the visual effects, we propose an $\alpha$-modulus lexicographical order where the first component is given by a function of ordering $I$,

$$
\begin{gathered}
I(p)=\beta\left[\kappa_{1} f_{R}(p)+\kappa_{2} f_{G}(p)+\kappa_{3} f_{B}(p)\right]+ \\
(1-\beta)\left[\max \left(f_{R}(p), f_{G}(p), f_{B}(p)\right)-\right. \\
\left.\min \left(f_{R}(p), f_{G}(p), f_{B}(p)\right)\right], 0 \leq \beta \leq 1 .
\end{gathered}
$$

In the function $I$ there are a linear combination of RGB components (i.e., a luminance value) and the $\max -\min$ of the components (i.e., a saturation value), weighted by $\beta$. After deep tests, we have found that the values $\kappa_{1}=$ $0.3, \kappa_{2}=0.6, \kappa_{3}=0.1$ and $\beta=0.8$ yield very good visual effects. Due to the fact that the luminance gives much importance to the green component, the other components for ordering can be: the red, then the green and finally, the blue $\left(I, f_{R}, f_{G}, f_{B}\right)$. A similar approach has been used for the interpolation of color images [6]. The order is called $I-R G B_{\alpha}$ and in Figure 1 are shown two examples of color leveling with this order.

The more homogenous HLS 3D-polar coordinate color representation may be used to define other interesting lexicographical orders. For this space, we adopted the lattices introduced in [3]. The hue is an angular component, therefore in order to be able to define a total order we must choice a hue origin $H_{0}$ (typically associated to the dominant color), i. e. using only the hue, $p<q$ if $f_{H}(p)+H_{0}>f_{H}(q)+H_{0}$. Having this constraint, we can define two $\alpha$-modulus lexicographical orders: luminancebased $L S H_{\alpha, H_{0}}$ (luminance, saturation and centred hue) and saturation-based $S L H_{\alpha, H_{0}}$ (saturation, luminance and centred hue). We have implemented also a lexicographical order with the hue component in the first level however, as pointed out in [3], the hue-based order is very unstable. The authors proposed a solution which is based on a weighting of the hue by the saturation, i.e. $f_{H}^{\prime}(p)=f_{H}(p)-H_{0}$ if $f_{H}(p)-H_{0} \geq 0^{\circ}$ or $f_{H}^{\prime}(p)=360^{\circ}+f_{H}(p)-$ $H_{0}$ if $f_{H}(p)-H_{0}<0^{\circ}$ and the following weighting, $f_{H}^{\prime \prime}(p)=\sup \left(f_{H}^{\prime}(p), 90\left(1-f_{S}(p)\right)\right), 0 \leq f_{H}^{\prime}(p)<90$ or $f_{H}^{\prime \prime}(p)=\inf \left(f_{H}^{\prime}(p), 90\left(1+f_{S}(p)\right)\right), 90 \leq f_{H}^{\prime}(p)<180$ or $f_{H}^{\prime \prime}(p)=\sup \left(f_{H}^{\prime}(p), 90\left(3-f_{S}(p)\right)\right), 180 \leq f_{H}^{\prime}(p)<270$ or $f_{H}^{\prime \prime}(p)=\inf \left(f_{H}^{\prime}(p), 90\left(3+f_{S}(p)\right)\right), 270 \leq f_{H}^{\prime}(p)<$
360. Now it is possible to define the hue-based order $H^{*} S L$, with the hue weighted value $f_{H}^{\prime \prime}(p)$ as first component $\left(p<q\right.$ if $f_{H}^{\prime \prime}(p)>f_{H}^{\prime \prime}(q)$, then the saturation and then the luminance). The application of $L S H_{\alpha, H_{0}}$ yields the best visual effects and consequently it is the most indicated for coding. The use of the saturation as priority for ordering can be interesting for feature extraction, segmentation, etc. [5] but the visual effects are annoying. The use of the $H^{*} S L$ results in inconvenient visual artefacts, mainly due to the fact that the influence of the choice for a dominant color is very important. This last lattice may be interesting for emphasising a particular color or for removing color regions but hardly for coding.

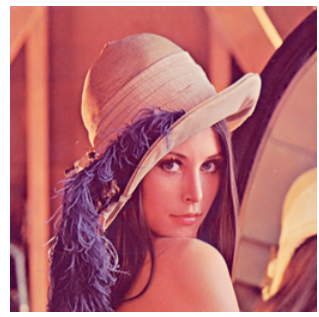

(a)

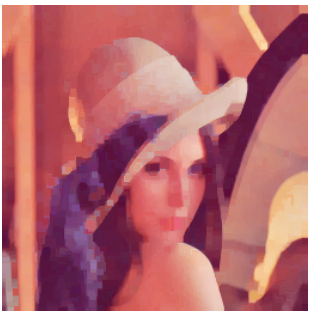

(b)

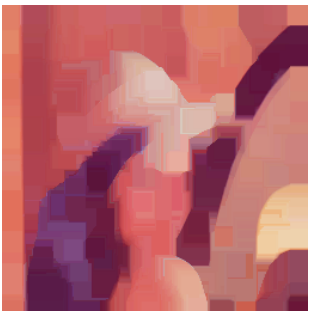

(d)

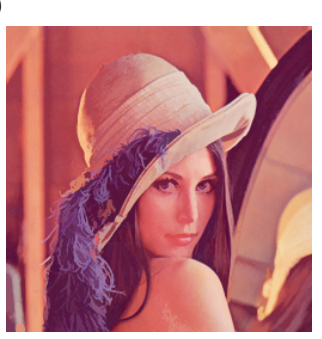

(c)

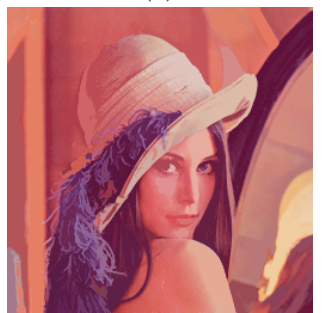

(e)
Fig. 1. Example of color levelings using the lexicographic order $I-R G B_{\alpha=10}$ on Lenna image: (a) Reference image, f. (b) Marker (ASF of size 5), $\mathbf{m}_{1}$. (c) Leveled image, $\Lambda\left(\mathbf{f}, \mathbf{m}_{1}\right)$. (d) Marker (ASF of size 15$), \mathbf{m}_{2}$. (e) Leveled image, $\Lambda\left(\mathbf{f}, \mathbf{m}_{2}\right)$.

\section{ALGORITHMIC FRAMEWORK}

Once these orders have been defined, the morphological operators are defined in the standard way. The vector erosion of a color image $\mathbf{f}$ at pixel $x$ by the structuring element $B$ 
of size $n$ is

$$
\varepsilon_{n B}(\mathbf{f})(x)=\left\{\mathbf{f}(y): \mathbf{f}(y)=\inf [\mathbf{f}(z)], z \in n\left(B_{x}\right)\right\},
$$

and the corresponding dilation $\delta_{n B}$ is obtained by replacing the inf by a sup. An opening $\gamma_{n B}$ is an erosion followed by a dilation, and a closing $\varphi_{n B}$ is a dilation followed by an erosion. A leveling takes as arguments two images, a reference function $\mathbf{f}$ and a marker function $\mathbf{m}$ (generally, the marker is a roughly simplified version of the reference image). The implementation of the leveling $\Lambda(\mathbf{f}, \mathbf{m})$ has been programmed by means of an iterative algorithm with geodesic dilations and geodesic erosions until idempotence [7, 2], i.e. $\Lambda(\mathbf{f}, \mathbf{m})^{i}=$ $\sup \left\{\inf \left[\mathbf{f}, \delta^{i}(\mathbf{m})\right], \varepsilon^{i}(\mathbf{m})\right\}$, until $\Lambda(\mathbf{f}, \mathbf{m})^{i}=\Lambda(\mathbf{f}, \mathbf{m})^{i+1}$. In order to have auto-dual levelings the marker function must be auto-dual. We have used two families of auto-dual markers: the alternate sequential filters (ASF), $A S F(\mathbf{f})_{n B}=\varphi_{n B} \gamma_{n B} \cdots \varphi_{2 B} \gamma_{2 B} \varphi_{B} \gamma_{B}(\mathbf{f})$ and the vector median filters (VMF), where the output corresponds to the color vector that minimises the sum of distances in the neighbourhood $n B$ [1]. The purpose of this study has been the comparison of the filters obtained for the five orders above presented, therefore we have programmed a generic "vector preserving" function for every morphological color operator (using optimal algorithms for the basic erosion/dilation). In the corresponding operator, an input variable allows to define the max and min of two color pixels according to the lexicographical order used. In conclusion, for each considered ordering we need only to build two additional max / min functions.

\section{EXPERIMENTAL RESULTS}

Apart from the visual effects (a subjective evaluation) of the different lexicographical orders discussed above, an objective evaluation of the obtained levelings has been carried out. Five color images have selected and for every image, six levelings have been computed using as markers: three $A S F$ 's of size $n=5,10$ and 15 ( $B$ is an square) and three $V M F$ of size $7 \times 7,11 \times 11$ and $11 \times 11$ applied two times. Moreover, this series of filters has been obtained for an example of each of the presented five lexicographical orders (notice that the $V M F$ markers, obtained from the RGB components, are always the same). For the $\alpha$-modulus lexicographical orders, the value of $\alpha=10$ has shown to achieve robust and nice levelings. The origin of the hue has been imposed to $H_{0}=0^{\circ}$. Then for every pair of initial image / leveled image, two parameters of quality have been calculated: the Signal-to-Noise Ratio $S N R$ and the Percentage of Reduction in the Number of Flat Zones $F Z R$ (connected components of the image with constant color value). In Tables 1 and 2 are included the average values on the six images for $S N R$ and $F Z R$. As we

\begin{tabular}{c|ccccc}
\hline $\mathbf{A S F}, n=5$ & $\Omega_{1}$ & $\Omega_{2}$ & $\Omega_{3}$ & $\Omega_{4}$ & $\Omega_{5}$ \\
\hline$S N R(\mathrm{~dB})$ & 21,9 & 22,1 & 22,4 & 17,4 & 17,8 \\
$F Z R(\%)$ & 37,1 & 37,5 & 38,0 & 40,0 & 39,4 \\
\hline \hline ASF,$n=10$ & $\Omega_{1}$ & $\Omega_{2}$ & $\Omega_{3}$ & $\Omega_{4}$ & $\Omega_{5}$ \\
\hline$S N R(\mathrm{~dB})$ & 18,3 & 18,7 & 19,1 & 15,5 & 14,3 \\
$F Z R(\%)$ & 44,3 & 44,8 & 45,3 & 46,4 & 46,4 \\
\hline \hline $\mathbf{A S F}, n=15$ & $\Omega_{1}$ & $\Omega_{2}$ & $\Omega_{3}$ & $\Omega_{4}$ & $\Omega_{5}$ \\
\hline$S N R(\mathrm{~dB})$ & 16,6 & 16,7 & 17,2 & 13,9 & 14,3 \\
$F Z R(\%)$ & 48,9 & 49,8 & 50,1 & 51,7 & 49,6 \\
\hline
\end{tabular}

Table 1. Average values of $S N R$ and $F Z R$ using $A S F$ as markers for the levelings and where $\Omega_{1}=M m-R G B$,

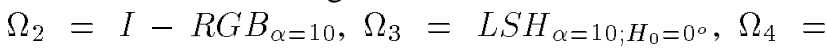
$S L H_{\alpha=10 ; H_{0}=0^{\circ}}$ and $\Omega_{5}=H^{*} S L_{H_{0}=0^{\circ}}$.

\begin{tabular}{c|ccccc}
\hline $\mathbf{V M F}_{7 \times 7}$ & $\Omega_{1}$ & $\Omega_{2}$ & $\Omega_{3}$ & $\Omega_{4}$ & $\Omega_{5}$ \\
\hline$S N R(\mathrm{~dB})$ & 23,8 & 24,1 & 24,0 & 22,3 & 22,2 \\
$F Z R(\%)$ & 33,6 & 34,1 & 31,0 & 26,9 & 31,1 \\
\hline \hline $\mathbf{V M F}, 11 \times 11$ & $\Omega_{1}$ & $\Omega_{2}$ & $\Omega_{3}$ & $\Omega_{4}$ & $\Omega_{5}$ \\
\hline$S N R(\mathrm{~dB})$ & 21,9 & 22,7 & 22,3 & 20,0 & 20,6 \\
$F Z R(\%)$ & 39,9 & 40,3 & 37,6 & 34,2 & 33,5 \\
\hline \hline $\mathbf{V M F}, 11 \times 11(\times 2)$ & $\Omega_{1}$ & $\Omega_{2}$ & $\Omega_{3}$ & $\Omega_{4}$ & $\Omega_{5}$ \\
\hline$S N R(\mathrm{~dB})$ & 20,8 & 21,4 & 20,9 & 18,9 & 19,4 \\
$F Z R(\%)$ & 44,9 & 45,3 & 43,2 & 38,5 & 37,8 \\
\hline
\end{tabular}

Table 2. Idem. using $V M F$ as markers for the levelings.

can observe, using the $A S F$ 's the best $F Z R$ corresponds to the lattices $S L H$ and $H^{*} S L$ which are however the worst lattices with respect to $S N R$. A good balance is given by the lattices $I-R G B$ and $L S H$, with results a little bit better for $L S H$. In the case of $V M F^{\prime}$ 's, owing to the fact that the medians are computed in the RGB space, the values of $S N R$ and $F Z R$ are a little better for $I-R G B$ although the values for $L S H$ are clearly upper than for $S L H$ or $H^{*} S L$.

\section{APPLICATION: IMPROVED COMPRESSION}

The most widespread image compression algorithms as JPEG (or MPEG for video sequences) are based on a transform coding scheme. It partitions an image into blocks, computes the discrete cosine transform (DCT) of each block and codes each DCT component according to a quantization scheme as a function of the magnitude of the component. The compression is greatest for constant or slowly varying blocks since these can be described by just a few DCT components. The best color levelings may be useful for the preprocessing of images for JPEG compression. The idea is to simplify the original image as much as possible without losing meaningful content. Obviously, the size of the removed structures is given by the size of the marker function used. Figure 2 depicts an example of this approach using a 


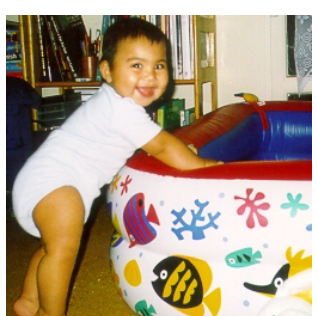

(a)

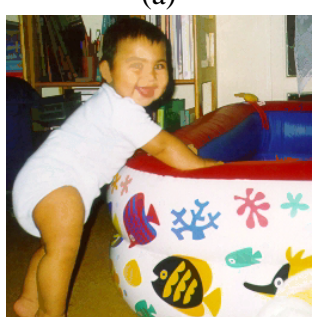

(c)

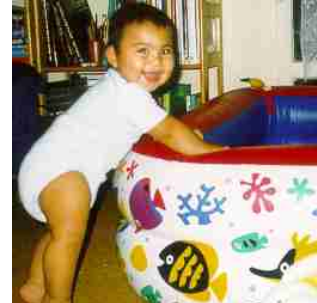

(b)

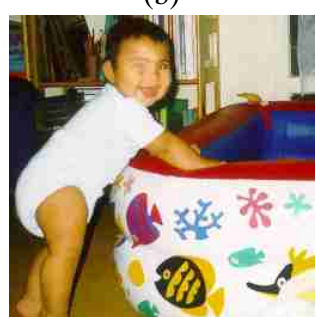

(d)
Fig. 2. Application to compression by JPEG on Carmen image: (a) Initial image (raster file 196662 bytes), (b) compressed initial image with quality $20 \%$ (6477 bytes), (c) leveled image, (d) compressed leveled image with quality $20 \%$ (5742 bytes).

leveling on $L S H_{\alpha=10}$ with a $V M F_{11 \times 11}$. In the leveled image, small details have been removed (see the black pupils of baby Carmen and the letters on the books) but, in the compressed initial image these details become diffused and therefore their suppression may be useful in order to have a clear image. The most interesting is the size reduction of $13 \%$ when applying the compression on the leveled image. For an image more abundant in details, the size reduction can be really significant; for instance the same leveling and the same compression quality applied to the standard $\mathrm{Ba}$ boon image yield a reduction of $30 \%$ and for the leveling of Lenna image from Figure 1(e) the reduction is upper than $35 \%$.

\section{CONCLUSION}

We discussed the use of lexicographical orders in the RGB and HLS color spaces for the implementation of vector levelings. It can be concluded that the most interesting lattice for morphological simplification and coding of color images is $L S H_{\alpha=10}$ (luminance, saturation, centred hue) since besides nice visual effects, it yields a good performance with regard to the $S N R$ and the enlargement of flat zones. We showed the performance of vectorial levelings for improving the JPEG compression of color images.

\section{REFERENCES}

[1] J. Astola, P. Haavisto and Y. Nuevo, "Vector Median Filters," in Proc. of the IEEE, Vol. 78, No. 4, pp. 678689, 1990.

[2] C. Gomila and F. Meyer, "Levelings in Vector Spaces," in Proc. of IEEE Conference on Image Processing, Kobe, Japan, October 24-28, 1999.

[3] A. Hanbury, "Mathematical morphology in the HLS colour space," in Proc. 12th BMVC, British Machine Vision Conference, Manchester, 10-13 September 2001, pp. II-451-460.

[4] A. Hanbury and J. Serra, "Mathematical morphology in the CIELAB space," Image Analysis and Stereology, 21, 201-206, 2002.

[5] A. Hanbury and J. Serra, "A 3D-polar coordinate colour representation suitable for image analysis," submitted to Computer Vision and Image Understanding, November 2002, 39 p.

[6] M. Iwanowski and J. Serra, "Morphological interpolation and color images," in Proc. of ICIAP'99, Venice, Italy, September 27-29, 1999.

[7] F. Meyer, "The levelings," in (H. Heijmans and J. Roerdink Eds.) Mathematical Morphology and Its Applications to Image Processing, pp. 199-206, Kluwer, 1998.

[8] F. Meyer, "Vector levelings and flattenings," in (J. Goutsias, L. Vincent and D.S. Bloomberg Eds.) Mathematical Morphology and Its Applications to Image Processing, pp. 51-60, Kluwer, 2000.

[9] F. Ortiz, F. Torres, J. Angulo and S. Puente, "Comparative study of vectorial morphological operations in different color spaces," in Proc. SPIE Algorithms, Techniques and Active Vision, Vol. SPIE 4572, pp. 259268, 2001.

[10] J. Serra, "Image Analysis and Mathematical Morphology. Vol I," and "Image Analysis and Mathematical Morphology. Vol II: Theoretical Advances," Academic Press, London, 1982 and 1988.

[11] J. Serra, "Anamorphoses and Function Lattices (Multivalued Morphology)," in (E. Dougherty Ed.), Mathematical Morphology in Image Processing, Marcel Dekker, 483-523, 1992.

[12] F. Zanoguera and F. Meyer, "On the implementation of non-separable vector levelings," in (H. Talbot and R. Beare Eds.) Mathematical Morphology, Proc. of ISMM'02, pp. -, Sydney, Australia, April 2002, CSIRO Publishing. 